\title{
Simulation of Past Life: Controlling Agent Behaviors from the Interactions between Ethnic Groups
}

\author{
Chen Kim Lim* ${ }^{* \ddagger}$, Marie-Paule Cani ${ }^{\dagger \ddagger}$, Quentin Galvane ${ }^{\dagger \ddagger}$, Julien Pettre ${ }^{\ddagger}$ and Abdullah Zawawi Talib* \\ ${ }^{*}$ School of Computer Sciences, Universiti Sains Malaysia, 11800 USM Penang, Malaysia \\ Email: azht@cs.usm.my \\ $\dagger$ LJK Laboratoire Jean Kuntzmann, Universite de Grenoble \\ ¥INRIA \\ Email:\{chen-kim.lim, marie-paule.cani, quentin.galvane, julien.pettre\}@inria.fr
}

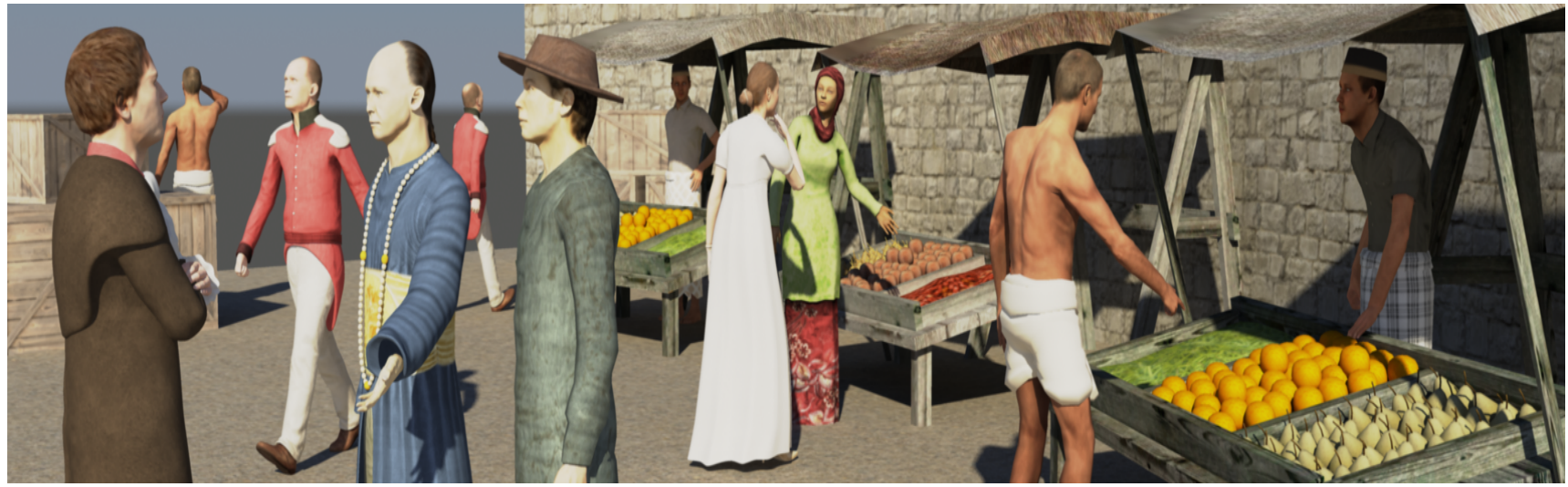

Fig. 1. Various trading interactions between diverse ethnic groups.

\begin{abstract}
Many efforts have been carried out in preserving the history and culture of Penang and also other regions of Malaysia since George Town was elected as a UNESCO living heritage city. This paper presents a method to simulate life in a local trading port in the $1800 \mathrm{~s}$, where various populations with very different social rules interacted with each other. These populations included Indian coolies, Malay vendors, British colonists and Chinese traders. The challenge is to model these ethnic groups as autonomous agents, and to capture the changes of behavior due to inter-ethnic interactions and to the arrival of boats at the pier. Agents from each population are equipped with a specific set of steering methods which are selected and parameterized according to predefined behavioral patterns (graphs of states). In this paper, we propose a new formalism where interactions between the different ethnics groups and with the boats can be either activated globally or locally. Global interactions cause changes of states for all the agents belonging to the target population, while local interactions only take place between specific agents, and result in changes of states for these agents only. The main contributions of our method are: i) Applying microscopic crowd simulation to the complex case of a multi-ethnic trading port, involving different behavioral patterns; ii) Introducing a high-level control method, through the interethnic interactions formalism. The resulting system generates a variety of real-time animations, all reflecting the adequate social behaviors. Such a system would be particularly useful in a virtual tour application.
\end{abstract}

\section{INTRODUCTION}

Three-dimensional (3D) modeling of ancient sites, cities or historical places has been widely applied to cultural heritage applications. Many fascinating applications were developed, providing impressive user immersion based on digital mockups. However, most digital cities are modeled without any inhabitant: users get the impression of visiting deserted environments, and cannot learn anything about the way the place was used in the past for daily life activities. Therefore, the next challenge is to enhance digital reconstruction of historical sites with animated populations, illustrating ancient or past life in these historical places.

In the task of digitally giving life to ancient or past populations, we believe that the main objective should be to re-create the general atmosphere of the place. The distribution of individual activities, the interactions between agents and with their surroundings, and the global motion that emerges from everyone's actions, create this atmosphere. Although little information may be available on how the population of these ancient or historical sites looked like and on the general impression that emerged from it, we can hardly find precise descriptions of the individual activities and of social interactions between people. In this paper, we propose to re-create past life from this perspective: we let the global atmosphere emerge from a detailed modeling of individual activities and of interactions between people. We therefore adopt a bottom-up approach, based on a microscopic crowd 
simulation technique which support more complex interactions through an action selection mechanism.

Microscopic crowd simulation avoids the laborious process of individually animating characters; they behave according to a simulation model. It is also based on the principle which states that the global-macroscopic-behavior of a crowd emerges from the local interactions between individuals: how each of them influences others and how these interactions are combined. It is thus a relevant approach to re-create populations in historical places, especially for frequently visited sites. Crowd simulation is already used to animate historical cities such as Rome [1] or Nicosia [2]. Even though they are based on microscopic approaches, the simulation is also driven by some global descriptions. For instance, in [1], users can directly describe which social status is expected for people in specific places. This is expressed through a semantic description of the city. The characters selected their goals based on this semantic. Their behaviors are locally influenced by this semantic too. Meanwhile, the interactions between characters are the same, whatever the place, and whatever their social status. In this work, we are tackling the problem of driving the changes of goal and behavior, for each virtual agent, from the multi-ethnic interactions taking place around it.

The World Heritage List includes 981 cultural or natural heritage places that are considered as having outstanding universal value [3]. This list comprises 759 cultural, 193 natural and 29 mixed properties in 160 States Parties and was ratified by 190 States in September 2012. In this paper, we attempt to recreate complex lively scenes for one of these historical places. We chose the trading port at Weld Quay in George Town, Penang, Malaysia (included in the World Heritage list from 2008) because it served as a meeting point, in the 19th century, for several types of population of very different origin and culture. Weld Quay was the earliest trading port during the colonial era of Malaysia. It was very famous for its strategic location, on the largest island encountered on the trading sea-route. Over the years, the exchange of human values and the mixture of cultures among many races living in this part of the city were well preserved. These make the port a unique and outstanding cultural area. However, with Penang being one of the topmost tourist destinations, Weld Quay still plays a leading role in the nation's shipping industry: the old trading port has transformed into a terminal for international cruise vessels. In order to construct the terminal, a number of captivating monuments and beautiful houses were demolished. There are not many works done on the virtual preservation and restoration of this site, in an effort to preserve, protect, and understand its specific cultural heritage and historical importance.

Although we do not disregard visual documents depicting past life at this trading port in general, we simulated the crowd mainly based on our rudimentary knowledge about the groups of people of different ethnicity, culture, language, and religion interacting there in the past for trading purposes: The different groups of people include English colonists and soldiers, the Malay vendors, the Indian coolies and the Chinese traders. This paper presents our method of modeling of the interactions between these diverse populations, and with the fleet of boats stopping at the port. Each population is associated with a set of possible states which corresponds to specific behaviors. The

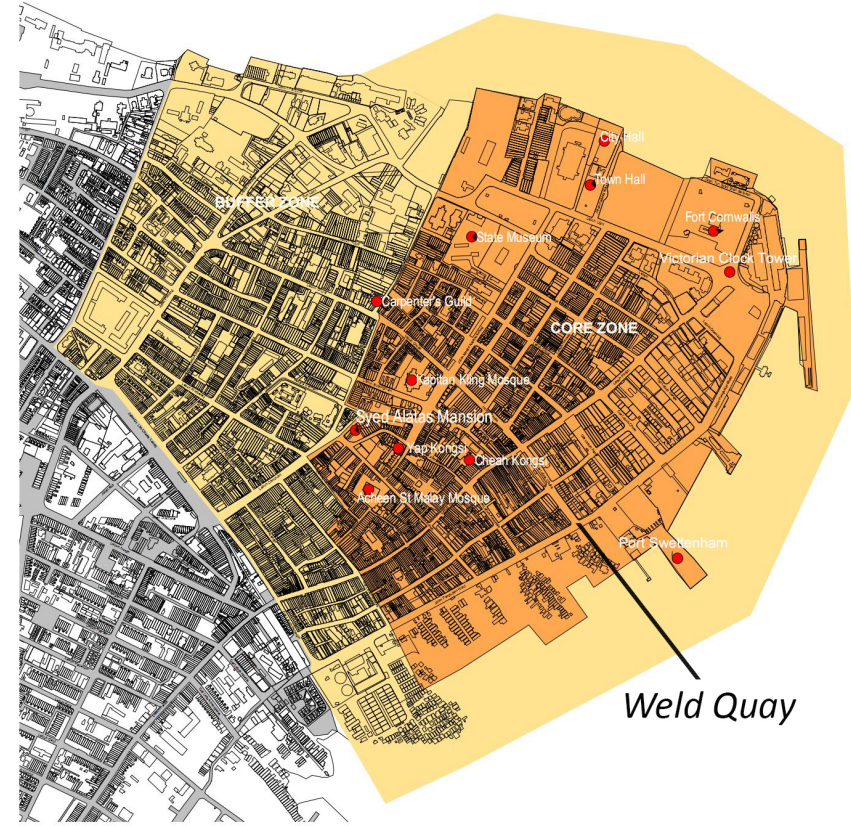

Fig. 2. The actual map of George Town heritage centre [4].

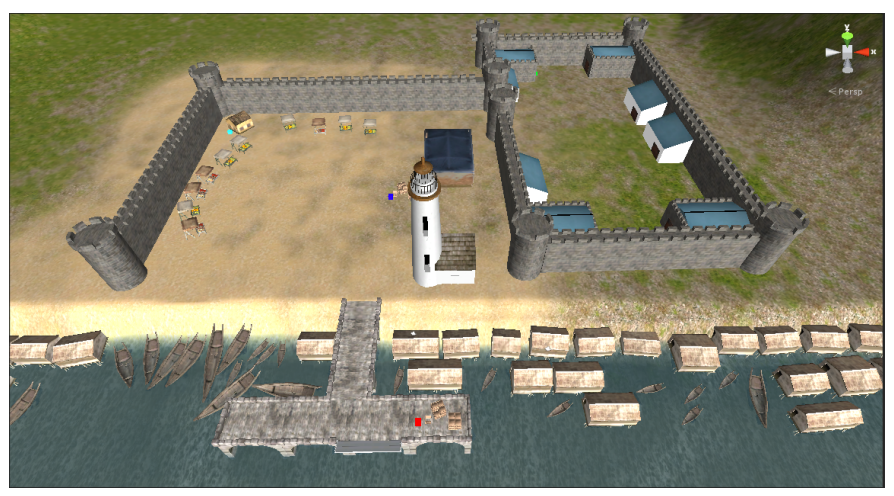

Fig. 3. The virtual environment of Weld Quay.

changes of states are triggered by global or local interactions between populations. The animations we produced, such as the one depicted in Figure 1, can be used for enhancing users immersive experience, and improve their understanding of the life in an Asian trading port in the 1800s. A virtual visit of the past will then provide a pedagogic insight into Penang's scenes in the first-half of the 19th-century, a rich historical tapestry which we cannot see today.

Section 2 reviews related work. Section 3 describes cultural background of this UNESCO heritage site. Section 4 shows the use of customized human template that covers different ethnic groups. Our models for the different populations are described in Section 5. Section 6 explains how global and local interactions are used to drive the changes of states in these models. Section 7 details the group formations techniques used for the English soldiers in two typical situations. We present our results in Section 8 before concluding the paper. 


\section{RELATED WORK}

Simulating agents' displacement and interactions in a plausible way is a challenging goal in crowd modeling systems. One popular approach, namely microscopic crowd simulation, consists in modeling the peoples as particles that interact with their environments through a set of applied forces. A wellknown example of such model is Helbing's social forces [5]. Reynolds pioneer work on flock simulation combined various interaction forces: cohesion, separation and alignment forces [6]. The method was later extended to a large variety of steering behaviors [7], [8], [9], such as repulsive bound, following wall, containment, obstacle avoidance, path following, queuing, seeking or pursuing, fleeing or evading, arriving and wandering. These types of approach are relevant in our case since various populations perform individualized actions and have diversified interactions.

The ViCrowd model [10] was used for simulating individuals and groups of people with various levels of realism including scripted, reactive and guided behaviors. Similarly, in this work, we control the crowd behaviors through innate and scripted behaviours, defining behavioral rules, using event and reactions and providing some external control to guide crowd behaviors. However, we focus on parameter adaptations for each agent, based on interactions, and apply them to different ethnics groups with evolving goals, instead of modeling different levels of autonomy with the same goal.

Particle-based crowd simulation models were extended by Tasse et al. [8] to model crowds walking in narrow ways and gathering around street performances in an African markets. Here, depending of its environment, each individual was able to switch between a set of predefined states. However, no mechanism was proposed to handle the interactions between different groups of peoples. The interaction between two populations of different nature was studied by Rodriguez et al. [9], with the simulation of pursuit and evasion behaviors on a complex terrain. Contrary to our goal, each of the populations is assigned with constant behaviors over time.

Other recent work focused on using the personality trait theory to simulate heterogeneous crowds where each individual is assigned with specific behavioral parameters [11]. Similarly, in this work, we allow some varieties within each population, but we combine this with changes of state and propose a formalism to drive the latter through global or local interactions with other population groups.

Other applications of crowds in cultural heritage applications include the simulation of demolished Pennysylvania Station in New York City, with mostly reactive behaviors [12]. In [13], the paper mainly focuses on individual behaviors and path-planning as some districts in Pompeii are restricted to a certain class of people, but not on various interactions among different populations. In [14], CrowdBrush is used to facilitate the design and their work does not focus on simulating the crowd. Bogdanovych et al. has simulated past inhabitants in the city of Uruk where the behaviors are concentrated on eating, sleeping, working and communicating with one another [15]. On the other hand, in our simulation we focused on the activities which involve tradings with different kinds of populated crowds.
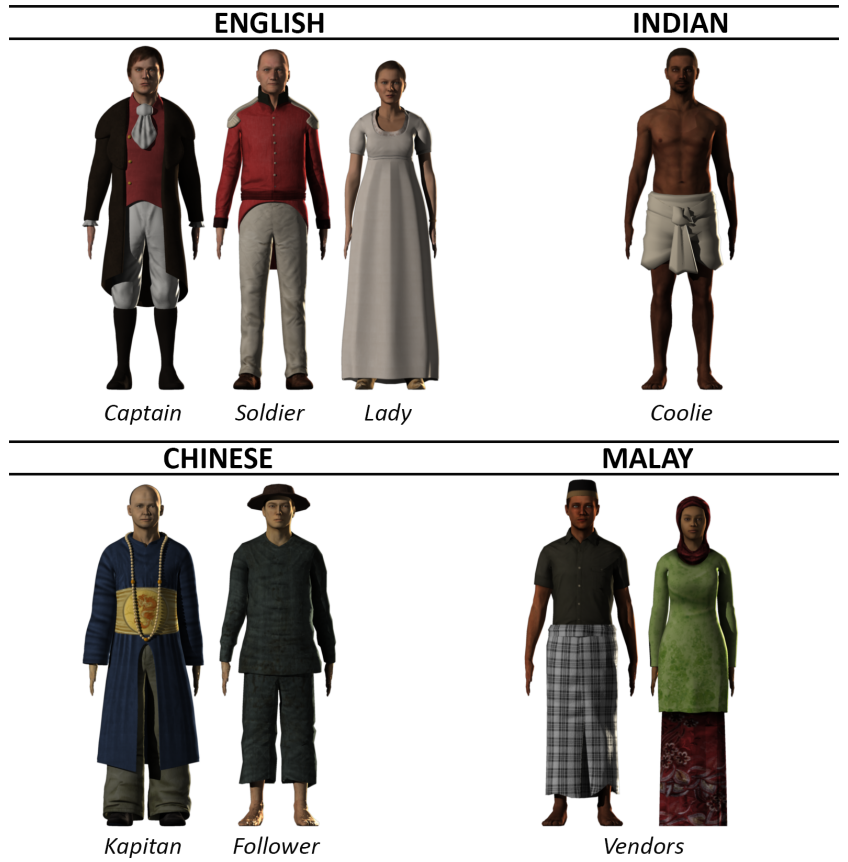

Fig. 4. The different ethnic groups represented in our simulation. Note the difference in types of traditional clothes.

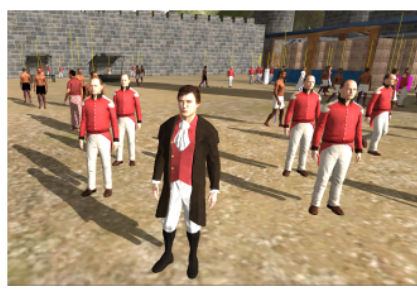

(a)

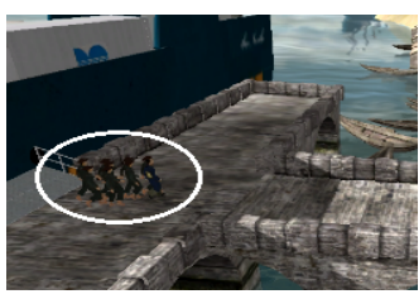

(c)

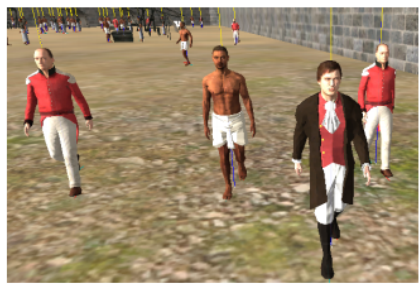

(b)

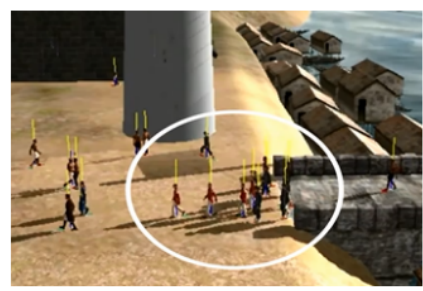

(d)
Fig. 5. (a) The English captain leads four soldiers side-by-side to the pier (b) A police catches a thief and requests for other colleagues to watch-out the thief by the sides. (c) The Chinese kapitan leads the Chinese workers. (d) The English troop escorts the Chinese group.

\section{Cultural BACKGROUND}

The history of modern Penang began when the island was leased to Captain Francis Light, an English trader-adventurer in exchange for military protection. For Light, Penang was a convenient place to build warehouses for trading purposes and an ideal location to curtail French expansion in Indochina and the Dutch foothold in Sumatra. On 11 August 1786, Francis Light landed on Penang, which was later called Fort Cornwallis. This occasion marked the beginning of more than a century of British involvement in Malaysia. The growth in the spice economy had triggered the movement of Chinese pioneers and traders to the island, which was actively encouraged by 
the English. However, in order to overcome the severe labor shortages during the era, the colonial administration began the practice of employing Indian convict workers as low-cost laborers or coolies. Archaeological evidence shows that Penang was also inhabited by the Semang-Pangan of the Juru and Yen lineages, both are now considered extinct cultures. They were hunter-gatherers of the Negrito stock having short stature and dark complexion, and were dispersed by the Malays as far back as 900 years ago. Since then the Malays were considered as the natives and owned local plantations, selling their local products for their living.

Sporadically located along Fort Cornwallis are old warehouses, called godowns, from the time when cargo was brought from the piers for temporary storage. On this southern stretch and on its off-shoot streets, you find various trades connected to the harbor, such as that of boats, engine repair, anchor making, and so on. The reclamation of the eastern shore that took place between 1883 and 1889 created a new coastal land for a deeper port is what is now called Weld Quay. The laying out of Weld Quay has progressed over year and the area has turned into a Chinese trading neighborhood, an area of wholesalers and Chinese warehouses as illustrated in Figure 2 . The neighborhood provided jobs for laborers and stevedores who developed their own living quarters, today known as the clan Jetties. The buildings along Weld Quay are originally numbered from the north to the south, on the western side of the street while the eastern side has only the harbor, piers and waterfront.

However, Penang port's initial pre-eminence was later supplanted by the Singapore port owing to its superior geographical location. However, Penang remained an important feeder to Singapore - channeling the exports meant for global shipping lines by ocean-going liners which had bypassed other regional ports. The replacement of sailing vessels by steamships in the mid-19th century cemented Penang's secondary importance after Singapore.

The rapid population growth stemming from economic development created problems such as sanitation, inadequate urban infrastructure, transportation and public health. Although there were efforts to save and preserve the trading port as an integral part of the heritage of Penang, the Jetties have lost much of their authenticity as well as their raison d'etre. It was originally constructed to house the port laborers and indentured coolies who were too poor to find housing on land. Nowadays, the descendants of these laborers are no longer finding work at the Penang harbor, now that harbor work has been very much mechanized. Therefore, what is preserved is not the jetties as they were, but a shanty-town or water-borne ghetto. While many argue that they are a tangible heritage, its evolution is beyond recognition.

So, it is important that we model the past trading port as illustrated in Figure 3 and simulate the different ethnic groups to show the original lifestyle of the inhabitants as the children of today should be given the opportunity for better appreciation of the multi-cultural activities that has transpired in the past.

\section{PEOPlE ACTIVITIES AND VISUAL ASPECT}

Our goal is to model the different kinds of occupations each ethnic group had in the 1800s. At that time, the English

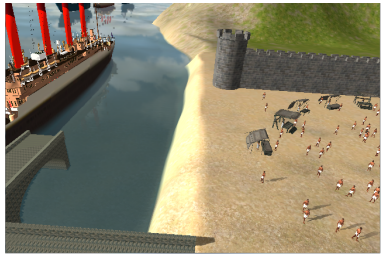

(a)

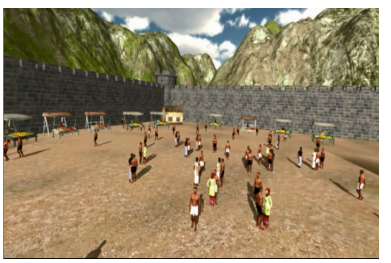

(c)

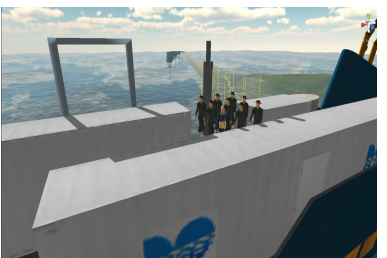

(e)

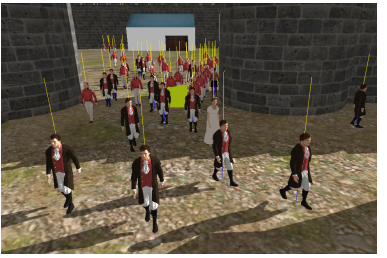

(g)

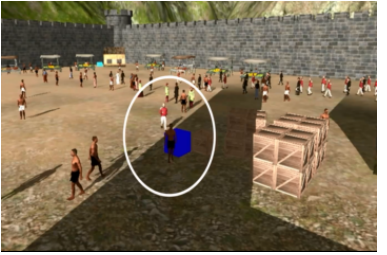

(i)

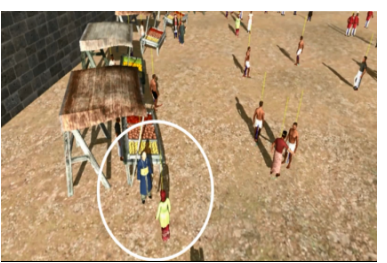

(k)

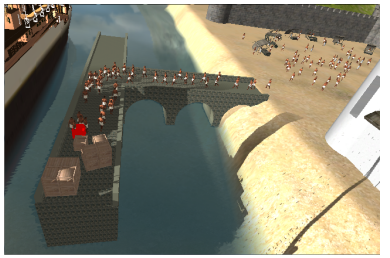

(b)

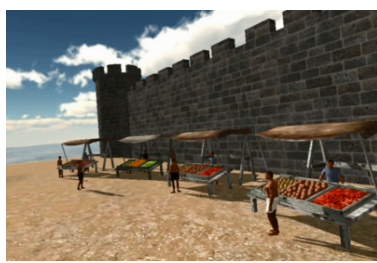

(d)

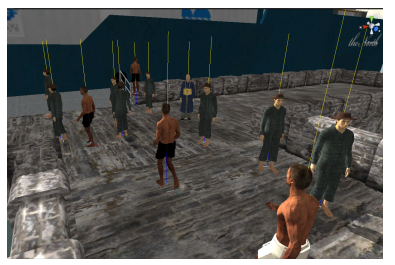

(f)

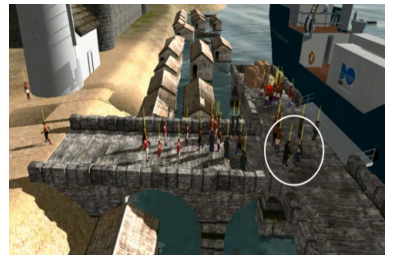

(h)

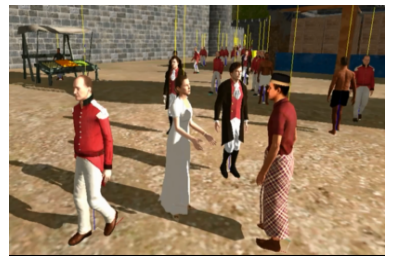

(j)

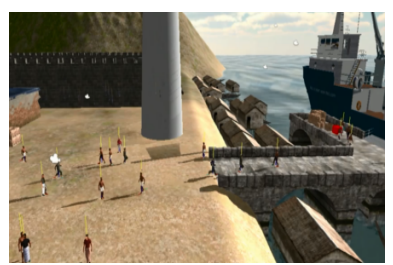

(1)
Fig. 6. (a) Two populations are first interacting and the boat is arriving. (b) Group of Indian coolies progressively switching from Reaching-Boat to Carrying behavior at the pier. (c) Indian potential buyers are targeted by Malays in Mobile-Selling state. (d) Some of the Malay vendors are looking after their stalls. (e) The Chinese traders are in the Standing state. (f) The traders are waiting at the pier to be escorted. (g) The English troop is exiting from the narrow path. (h) The English captain greets his customer. (i) The policeman is chasing a thief. (j) The English woman is busy haggling with the vendors over the price of goods. (k) The Chinese kapitan also involves himself in communication, argument and negotiation. (l) The Chinese traders leave hastily their current states and switch to Exiting state to sail away with the boat. 
colonists include the captain who oversees the fort, the soldiers who monitor the area around to ensure the security of the people, and the officer wives who spend most of their time haggling with vendors over the price of goods. The Chinese trader acts as an ambassador between China and the colony of Penang who traded their goods in this trading port. Both the Malay men and women are static and mobile sellers in the marketplace while the Indians brought in by the colonial administration are used for public services or work as laborers.

Being able to easily differentiate between these categories of people through visual clues is essential for understanding the range of activities taking place. Moreover, using plausible aspect and closing is important for a cultural heritage application. As in [1], we thus use different human models and costumes for the different ethnic groups. In our case, we relied on some depictions of the traditional costumes at this time period, and on other information found in [16].

The traditional costumes we used for each ethnic group are shown in Figure 4. The English colonists are dressed in British style, the Chinese traders are dressed in a uniform that has a dragon motif and keep long hair in plaits. They also wear a pearl necklace which shows their superiority over the workers who wear fishing hats and are bare-footed. The Malay man is dressed with sarong with mosaic patterns around their waist and songkok on the head that they wrap around their waist while the Malay woman is dressed in a fanciful and bright tudung which is a scarf on their head. The Indians are very well-built and dressed in a plain cloth or black or white wrapping around the lower part of their bodies which is called sash.

\section{Modeling the Ethnic Groups}

We use independent groups, each defined using the formalism in [6], to model different populations (i.e. different groups of agents) with specific sets of possible states. Within a group, each state activates a given set of steering forces and different ranges of parameter values. In our implementation, we currently use a standard set of possible forces. To avoid homogeneous behavior within a population, individual parameters are set at random within a range of possible values. To save computational time, we applied the Monte Carlo method described in [17] to compute the global steering forces while avoiding the costly re-evaluation of each steering force at each simulation step.

Let us take the example of our cultural heritage application. The boats stop at the pier and Indian coolies come to download the goods and carry them to large containers. Meanwhile, Malay vendors look for potential clients. They are either stationary at their stores or scattered around the port. When some of the Indian coolies have finished their work and started wandering around, the mobile Malay vendors approach them, to try to sell their goods. If they succeed, the individual Indian remains with the vendor for a while. The boat sails away as soon as all the goods have been downloaded. We model this scenario using five different groups as listed below (see also Figure 8). The interactions driving their changes of state will be described in Section VI.

Boats: Boats are modeled as particles with two different possible states: Resting or Sailing. The Resting state is used when the boat is at the pier where no force is applied. In the Sailing state, a path-following behavior is used thanks to a number of predefined way-points. In addition to the usual mass and maximum speed parameters, each boat is assigned with a Load parameter representing the amount of goods it currently carries.

Indians Coolies: The population of Indian coolies is given four different states: Resting, Wandering, Reaching-Boat, and Carrying-Goods. In the Wandering state, Indians are given the standard 'flocking' behavior [6] (cohesion, alignment and separation forces), in addition to obstacle avoidance behavior. They are set to remain in small groups thanks to a strong weight for the cohesion force. In the Reaching-Boat and Carrying-Goods states, they are assigned collision avoidance and path-following behaviors. The paths are used to lead respectively to the point where they load the goods at the pier and to the container where they download them, and vice versa. The mass of agents is increased and their maximum speed is decreased when they carry goods. To avoid the Indians all crowding at one way-point before trying to reach the boat, we provide different starting way-points and they use the closest way-point when they enter the Reaching-Boat state.

Malays Vendors: This local population travels towards the port to sell local food and products. While some individuals guard their stalls at the market place, others are more aggressive and spread out to approach the potential customers. We use three states to model them: Resting, Mobile-Selling, and Wandering. Resting, where no force is applied, is used to model static selling at the market place. The Mobile-Selling state corresponds to a target seeking behavior where the target is the closest available possible customer (an agent from a different population). The Wandering state is similar to the one for the Indians, except that we use different ranges of parameters to model less cohesiveness among the slowly and wandering groups of agents.

English Colonists: The English soldiers are initialized in the Wandering state where they monitor around the terrain to ensure the security. When a boat arrives at the pier, they switch to Reaching-Boat to greet Chinese customers. The English captain will lead the customers to the fort and the English soldiers demonstrate the path-following behavior by marching behind the captain. Both the captains and soldiers take part actively in running after the thief when there is a robbery occurring. The English instructs the thief to freeze when they manage to arrest the thief and bring him to prison in the Arresting state with the help of other colleagues, escorting from behind. In this case, the colleagues are taking the thief as their leader, while the soldier who caught the thief, is the leader of the thief. Otherwise, if the distance between the thief and the soldier is too large, the English soldier will stop chasing. The English women are only involved in the interaction with the Malay vendors to haggle over the price of goods.

Chinese Traders: This ethnic group sails from afar in the boat in the Standing state. When the boat arrives, the Chinese businessmen called kapitan waits for the English captain to approach him. After greeting has taken place, the kapitan alerts the rest of the workers to follow him. The kapitan is adapting the current target speed of the English captain in order to be walking in line with him. One of the workers switches to the Supervising state in order to look after the loading process at 
the container. When they arrived at the fort, they are back to the Wandering state and they can be involved in the interactions with Malay vendors. When the number of goods on the boat is null, the Chinese will be Exiting and board on the boat to sail away regardless of the current interactions they are having.

\section{INTERACTING POPULATIONS}

In our formalism, interactions between different populations are used as event-driven switches of states. These interactions can be either global or local, depending if they apply to the entire populations or to specific individuals as illustrated in Figure 8. We give below four examples of such interactions.

Boat-Indians coolies interaction: When a boat in the Sailing state approaches the pier way-point, a global interaction is used to switch all the Indians (except those in the GoodCarrying state) to the Reaching-Boat state. This models the fact that all the inactive coolies are waiting for the boat and immediately aware that the boat is approaching. In contrast, local interactions are used between a boat At-The-Pier and the Indians coolies: each Indian coolie passing through the loading way-point switches the state to Good-Carrying and decreases the Load parameter of the boat by one. The state of the agent is switched back to the Reaching-Boat state when they get to the container where the goods are downloaded. A boat with zero load switches back to the Sailing state and applies some global interactions again to the Indians coolies: all those in Reaching-Boat state switch to their default Wandering state.

Indians coolies-Malay vendors interaction: The Malay population is not affected by the boat but behaves according to the number of Indians coolies in the Wandering state. A global interaction for Indians coolies to Malay vendors enables the Malay vendors to be aware, at each simulation step of the current number of available Indian customers (those in Wandering state exclusively). If this number is non-zero, the closest Malay vendor in Mobile-Selling state assigned particular Indian as a target, and the number of available customers is decremented. If the Malay vendor approaches its target closer than a threshold, a local interaction is activated: both are switched to Resting state for a given number of steps to model the transaction that is taking place. Both Indian coolie and Malay vendor switch back to Wandering. Lastly, we added another distance-based local interaction: when Indian and Malay agents are closer than a safe distance, they are added to the list of obstacles, taken into account by the other for collision avoidance. This is required since these agents are managed by different population managers.

Malay vendor-English colonist interaction: When the mobile vendors try to interact and sell their local products to other ethnic groups, they might be robbed. The Malay vendor who is being robbed triggers a propagation of asking for help through a one-to-many interaction: all the English soldiers who were performing other local interactions are alerted when the robbery comes to light. The nearest soldier to where the robbery took place switches state and chases the thief. When he manages to catch the thief, he alerts his closest colleagues that he needs other helpers to escort the thief to the prison. As soon as the robbery case is handled by the English, the Malay vendor goes back to the approaching customers state.

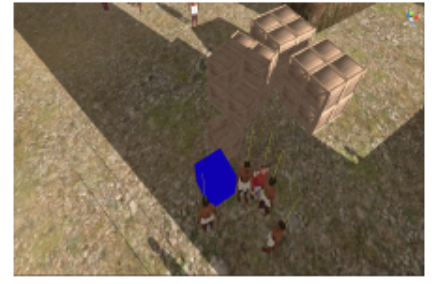

(a)

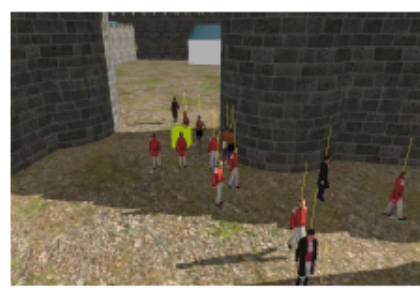

(c)

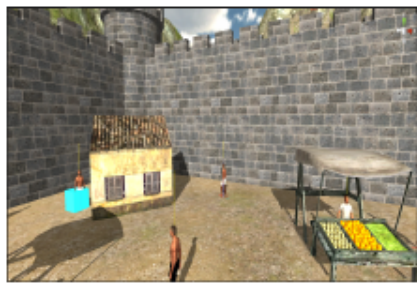

(e)

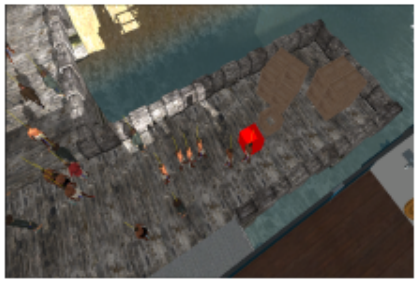

(b)

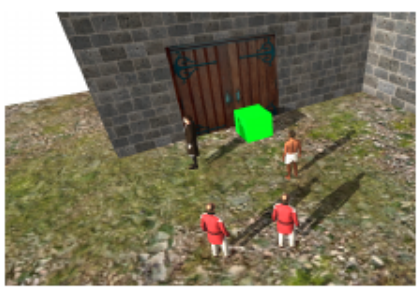

(d)

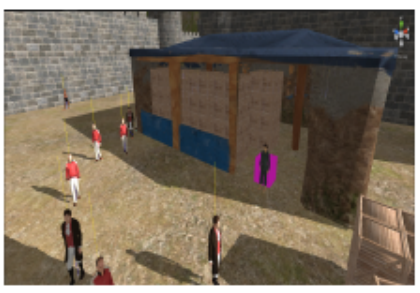

(f)
Fig. 7. (a) The Indian coolies are uploading the goods at the pier as indicated in red-colored way-point. (b) The Indian coolies carry the goods and download them at the container as indicated in bluecolored way-points. (c) Different ethnic groups arriving at the fort as indicated in yellow-colored way-point. (d) The thief arrives at the prison as indicated in green-colored way-point. (e) The thief character is suppressed and re-appears as a new character as indicated in light blue-colored way-point. (f) One of the Chinese workers is assigned to supervise the loading process at the container as indicated in purplecolored way-point.

Chinese traders-Boat interaction: The boat sails with the Chinese traders and loaded goods. When the boat arrives at the pier, the Chinese traders enter the pier and wait for English colonist to come, and welcome and escort them to the fort. When the Chinese traders arrive at the fort, they can perform local interactions with peoples in the market place. The Indian coolies continue to load the goods to the container until they are empty at the pier. When the goods are null, the boat alerts the Chinese traders to stop their current local interactions and get back to the boat. The Chinese traders receive the alert through this global interaction. They rush back to the boat and the boat sails away.

\section{GROUP FORMATIONS}

There are two main types of leader-following behaviors model implemented in this paper. Scenario 1: When an English leader walks towards the T-shaped pier to welcome his Chinese customer, a captain brings along four soldiers that are following behind him two-by-two in a proper alignment. The leader has four different offsets points based on the right offset and the forward offset scalars. This grouping results in a negated V-like formation between the leader and the followers with Line-Abreast formation among the soldiers behind the first formation as illustrated in Figure 5(a). 
Scenario 2: When an English catches a thief, the thief has to follow him to the prison after interrogation. He will ask for another two colleagues to escort the thief behind him until all of them reach the prison as the thief usually does not follow the policeman voluntarily. Note that both the captain and the soldier can be represented by the role of a policeman. The thief is following a single offset point of the policeman while becoming the leader to both the escorting English behind him. This grouping results in a River-like formation between the policeman and the thief and then a negated V-like formation behind the thief as shown in Figure 5(b).

A group of 2-3 persons has the most reasonable degree of freedom between each agents [18]. There is also a complex group formation from the group of English colonists and Chinese traders. The English soldiers are following their captain while the Chinese workers are following the Chinese kapitan. Figure 5(c) illustrated the leader who is followed by the Chinese group exiting from the boat. Each leader has its own way-point side-by-side until they reach the fort. This is inspired by the work of [19]. By having multiple way-points, it can be a simple solution to deadlock for a big group of 14 peoples as illustrated in Figure 5(d).

\section{RESULTS AND CONCLUSION}

The simple scenario we presented in this paper is our first attempt in modeling multi-cultural trading in Weld Quay, Penang in Malaysia, back in the 19th century [20]. The implementation was done using the Unity3D SDK [21]. Introducing different congregations for the boats and for four ethnic groups, together with global and local interactions between them to drive changes of states, enables us to get coherent animations conforming to our scenario, as illustrated in Figure 6, as well as in the accompanying video.

Our current model simulates the scene of Chinese traders arriving with the boats and trading goods with the English, customer of various ethnic groups at the market place, and complex interactions such as soldiers arresting a thief. In the simulated scenes there are goals for the agents to reach depending on the changes of states through the indication of the triggered way-points as illustrated in Figure 7. Future scenarios might include user controls. This will be other opportunities for us to test the expressiveness of our interaction model, driving changes of states. We would also like to have an animation bank where we store the captured motion and animation of "carrying goods", "running away from police" and so on, and matches with the switches of events.

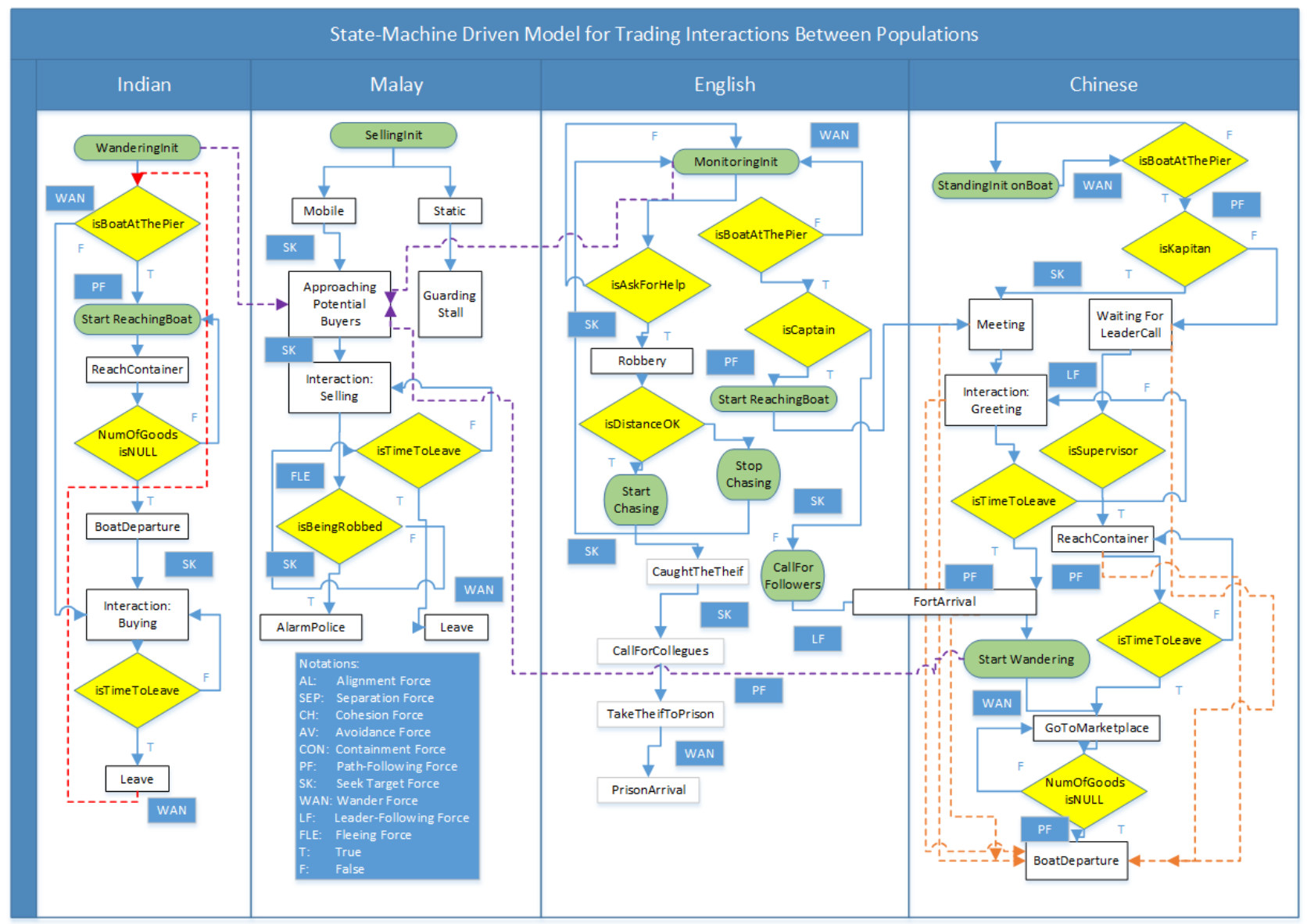

Fig. 8. The structure of the state machine-based model for the interacting diversified populations. The green ovals represent the states in which the agents switch between data-driven locomotion and the procedural motion model, the yellow diamonds represent decision makings for the different desired behaviors and the white squares represent types of interactions that changes their intentional goals. The red-dotted line indicates the routine of update per frame to switch from local interaction to global interaction if the boat is at the pier. The purple-dotted lines show a list of potential buyers for the vendors to approach and the orange-dotted lines indicate the rapid change of any states to boat departure when the goods have been downloaded. Note that there are always some steering forces applied strongly for each sets of events. 
To achieve our crowd simulation at interactive rates, we used a limited number of agents: considering the scale of the environment, we chose to animate 120 Indians coolies, 30 Malay vendors, 50 English colonists, 36 Chinese traders, and four boats, which is sufficient to validate the full scenario we just described. Although this relatively small number of agents makes the understanding of the activities easier, using about 2000 agents would give a more realistic representation of the way Weld Quay may have looked like. A solution to handle this complexity would be to switch to the crowd patches approach [22]. However, modeling different populations that change state over time within this formalism, where local, precomputed simulations are usually played in loop, would require new research contributions.

Crowd patches technique is a database approach which the assembly data are the patches for the expressiveness of the trajectories and capturing the animation. The problem is that the technique is currently less attentive in predicting avoidance of other patches for different ethnic groups. The solution is to diverge this technique to scenario-based for other motions that are behind the background where the camera is more focused on the simulated agents by making a clear distinction of the primary and secondary agents which switch from crowd patches to simulation from time to time.

Another future direction is on the selection of steering and interaction models as well as of their parameters. All these elements are combined to produce an animation. One difficult task for the designer is to make the right selection and tuning of models for the animation to follow a given scenario, or to reach a desired level of realism. These question needs to be addressed as for now, the quality of results are mainly based on the artistic skills of the designers.

\section{ACKNOWLEDGMENT}

Many thanks to French embassy in Malaysia and USM RESEARCH UNIVERSITY GRANT: 1001/PKOMP/817062. Part of this work was supported by the ANR project CHROME ANR-12-CORD-0013 and by the ERC advanced grant EXPRESSIVE ERC-2011-ADG-20110209.

\section{REFERENCES}

[1] D. Thalmann and S. R. Musse, Crowd Simulation, ser. Computer science. Springer-Verlag London Limited, 2007.

[2] M. Dikaiakou, A. Efthymiou, and Y. Chrysanthou, "Modelling the walled city of nicosia," in Proceedings of the 4th International conference on Virtual Reality, Archaeology and Intelligent Cultural Heritage, ser. VAST'03. Aire-la-Ville, Switzerland, Switzerland: Eurographics Association, 2003, pp. 61-70.

[3] http://whc.unesco.org/en/list.

[4] http://www.world-heritage-site.com/2011/06/05/about-george-townpenang/.

[5] D. Helbing and P. Molnar, "Social force model for pedestrian dynamics," Physical Review E, vol. 51(5), pp. 4282-4286, 1995.

[6] C. W. Reynolds, "Flocks, herds and schools: A distributed behavioral model," SIGGRAPH Comput. Graph., vol. 21, no. 4, pp. 25-34, Aug. 1987.

[7] C. Reynolds, "Steering behaviors for autonomous characters," 1999.

[8] F. P. Tasse, K. R. Glass, and S. Bangay, "Simulating crowd phenomena in african markets," in Afrigraph, 2009, pp. 47-52.
[9] S. Rodriguez, J. Denny, T. Zourntos, and N. M. Amato, "Toward simulating realistic pursuit-evasion using a roadmap-based approach," in $3 r d$ International Conference on Motion in Games, ser. MIG'10, 2010, pp. 82-93.

[10] S. R. Musse and D. Thalmann, "Hierarchical model for real time simulation of virtual human crowds," IEEE Transactions on Visualization and Computer Graphics, vol. 7, no. 2, pp. 152-164, Apr. 2001.

[11] S. J. Guy, S. Kim, M. C. Lin, and D. Manocha, "Simulating heterogeneous crowd behaviors using personality trait theory," in $A C M / E G$ Symposium on Computer Animation, ser. SCA '11, 2011, pp. 43-52.

[12] W. Shao and D. Terzopoulos, "Autonomous pedestrians," in Proceedings of the 2005 ACM SIGGRAPH/Eurographics symposium on Computer animation, ser. SCA '05. New York, NY, USA: ACM, 2005, pp. 19-28.

[13] J. Maïm, S. Haegler, B. Yersin, P. Mueller, D. Thalmann, and L. V. Gool, "Populating ancient pompeii with crowds of virtual romans," in Proceedings of the 8th International Symposium on Virtual Reality, Archaeology and Intelligent Cultural Heritage, D. Arnold, F. Niccolucci, and A. Chalmers, Eds., November 2007.

[14] B. Ulicny, P. d. H. Ciechomski, and D. Thalmann, "Crowdbrush: interactive authoring of real-time crowd scenes," in Proceedings of the 2004 ACM SIGGRAPH/Eurographics symposium on Computer animation, ser. SCA '04. Aire-la-Ville, Switzerland, Switzerland: Eurographics Association, 2004, pp. 243-252.

[15] A. Bogdanovych, K. Ijaz, and S. Simoff, "The city of uruk: teaching ancient history in a virtual world," in Proceedings of the 12th international conference on Intelligent Virtual Agents, ser. IVA'12. Berlin, Heidelberg: Springer-Verlag, 2012, pp. 28-35.

[16] Z. Sual, F. Azzam, P. Mohamad, and K. d. P. Malaysia. Kementerian Kebudayaan, Citra busana tradisional Malaysia. Kementerian Kebudayaan, Kesenian dan Pelancongan, Malaysia, 2002.

[17] J. Van den Berg, S. Patil, J. Sewall, D. Manocha, and M. C. Lin, "Interactive navigation of multiple agents in crowded environments," in Symposium on Interactive 3D Graphics and Games. ACM, 2008, pp. 139-147.

[18] M. Moussaïd, N. Perozo, S. Garnier, D. Helbing, and G. Theraulaz, "The walking behaviour of pedestrian social groups and its impact on crowd dynamics," PLoS ONE, vol. 5, p. e10047, 042010.

[19] N. Pelechano, B. Spanlang, and A. Beacco, "A framework for rendering, simulation and animation of crowds," in CEIG Spanish Conference on Computer Graphics, 2009.

[20] S. N. Khoo, More than merchants : a history of the German-speaking community in Penang, 1800s-1940s. Areca Books, 2006.

[21] http://unity3d.com.

[22] B. Yersin, J. Maïm, J. Pettré, and D. Thalmann, "Crowd patches: populating large-scale virtual environments for real-time applications," in Symposium on Interactive 3D graphics and games, ser. I3D '09, 2009, pp. 207-214. 